\title{
Die Konjunktur der qualitativen Sozialforschung und Konjunkturen innerhalb der qualitativen Sozialforschung ${ }^{1}$
}

\section{Jo Reichertz}

\section{Keywords:}

Berliner

Methodentreffen

Qualitative

Forschung;

Geschichte der

qualitativen

Sozialforschung;

Konjunktur;

kritische

qualitative

Forschung;

elaborierte

Forschung; Ad-

hoc-Forschung;

QDA; qualitative

Datenanalyse
Zusammenfassung: Der vorliegende Aufsatz untersucht die Geschichte der qualitativen Sozialforschung mit dem aus der Ökonomie entlehnten Begriff der "Konjunktur" und will zeigen, dass eine solche Perspektive sinnvoll ist. Es zeigt sich nämlich, dass innerhalb der qualitativen Sozialforschung nicht ein bestimmter Trend oder eine bestimmte Entwicklung auf ein Ziel hin zu beobachten ist, sondern ein Auf und Ab der Themen und Vorlieben. Zum Abschluss wird versucht, eine Bilanz des aktuellen Standes der qualitativen Sozialforschung zu ziehen.

\section{Inhaltsverzeichnis}

1. Die qualitative Sozialforschung hat Konjunktur

2. Erste Wertung

3. Die Konjunktur der qualitativen Forschung

3.1 Erfolgreich oder nicht?

3.2 Kanonisierung bei gleichzeitiger Zersplitterung

4. Konjunkturen der methodischen Ansätze

5. Konjunkturen der Themen

\section{$\underline{5.1}$ Gültigkeit}

\section{$\underline{5.2}$ Festschreibung von Offenheit}

5.3 Das Allgemeine oder das Besondere oder was?

$\underline{5.4}$ Triangulation

\subsection{Mixed Methods}

6. Weisen die Konjunkturen ein Muster auf?

6.1 Auf der Ebene des Forschungsgegenstandes

6.2 Auf der Ebene der Forschungspraxis

6.3 Auf der Ebene der Legitimation von Forschung

$\underline{6.4}$ Auf der Ebene der Adressat/innen von qualitativer Forschung

7. Was bewegt die verschiedenen Konjunkturen?

$\underline{7.1}$ Veralltäglichung des Charismas

7.2 Vom Reputationsmarkt zum ökonomischen Markt

7.3 Abschied von Therapie und Kritik

1 Danken möchte ich ganz herzlich Günter MEY dafür, dass er mich genötigt hat, im Rahmen der Mittagsvorlesung des $\underline{5}$. Berliner Methodentreffens Qualitative Forschung über Konjunkturen nachzudenken. Wie sich erst später für mich herausstellte, ist die Einnahme einer solchen Perspektive sehr fruchtbar. Bei den Überlegungen zum Stand der qualitativen Sozialforschung beziehe ich mich hier auf eine Debatte, die im Jahr 2007 in der Zeitschrift Erwägen, Wissen, Ethik (EWE 18, Heft 2) stattgefunden hat (REICHERTZ 2007a und 2007b). An der Debatte über Ansprüche, Prämissen und Probleme der qualitativen Sozialforschung beteiligten sich über 30 qualitativ arbeitende Forscher und Forscherinnen mit teils recht unterschiedlicher Ausrichtung. Insofern liefert dieses Heft einen einzigartigen Überblick über den derzeitigen Stand der Debatte vor allem innerhalb der deutschsprachigen qualitativen Sozialforschung. 
Literatur

Zum Autor

Zitation

\section{Die qualitative Sozialforschung hat Konjunktur}

Geschichte, auch die der qualitativen Sozialforschung, entwickelt sich nicht (einmal auf die Bahn geschickt) gradlinig, eine Stufe nach der anderen nehmend - auf ein Ziel hin gerichtet. Geschichte, also auch die der qualitativen

Sozialforschung, entwickelt sich stattdessen sprunghaft - dabei manchmal auch die Richtung wechselnd. [1]

Auch in der qualitativen Sozialforschung kommen und gehen Themen. Das gilt sowohl für die Methoden der Datenerhebung und Datenauswertung als auch für die Theoriebildung. Was gestern noch als zentral erachtet wurde, ist heute peripher, und das, was heute niemand nachfragt, wird morgen hoch gehandelt. [2]

Es gibt also auch in der qualitativen Sozialforschung ein "Auf und Ab" Konjunkturen. Qualitative Methoden haben nämlich nicht nur Konjunktur, sondern auch ihre Konjunkturen. Das Sprechen von "Konjunktur" ist nun die Inanspruchnahme einer Metapher, die erst einmal ganz harmlos daherkommt. Sie soll in der Regel etwas verdeutlichen - klarer machen. Aber Metaphern machen nicht nur etwas klarer, sie sind niemals harmlos, sie treffen immer auch Entscheidungen, sie sagen etwas Bestimmtes aus. Das gilt auch für die hier gewählte Metaphorik von der "Konjunktur". Damit einher geht nämlich bereits eine weitgehende und keineswegs unstrittige theoretische Positionierung im Hinblick auf den Erkenntnisfortschritt in der Wissenschaft: Wer von Konjunktur spricht, behauptet implizit, dass Wissenschaftler/innen Erkenntnis nicht Schritt für Schritt anhäufen, dass der Erkenntnisweg nicht zu einem "Immer mehr" und "Immer besser" führt, dass also Wissenschaftler/innen nicht langsam und beharrlich den gesellschaftlichen Erkenntniskübel auffüllen (POPPER 2004, S.369ff.), sondern wer von Konjunkturen spricht, spricht von einem Hin und Her des "Aufmerksamkeitsscheinwerfers" wissenschaftlicher Forschung, spricht eher von Paradigmenwechseln (KUHN 1976) als von Erkenntnisfortschritt, spricht im Falle der gewählten Metaphorik auch von Märkten. Das müssen nun nicht ökonomische Märkte sein, es können auch andere sein, wie z.B. der Geschmacks- oder Reputationsmarkt. [3]

Ein eigener "Markt" der Methoden sagt, was angesagt ist, was nachgefragt wird und was sich gut "verkauft". Dieser Markt entsteht und entwickelt sich durch die Handlungen, Interpretationen, Hoffnungen, Problemlagen, Gelegenheiten und Einschränkungen von individuellen wie kollektiven Akteur/innen im Feld der qualitativen Sozialforschung, die darauf hoffen, durch ihr Handeln ihre Probleme zu bearbeiten oder gar zu lösen. Handelnde in diesem Feld sind sowohl die Shareholders, also die, die mit ihrer Arbeitskraft und ihrer Reputation in diese "Aktie" investiert haben (Wissenschaftler/innen, Studierende, Institute), als auch die Stakeholders, also die, die direkt oder indirekt von der qualitativen 
Sozialforschung betroffen sind (Beforschte, Auftraggeber/innen, Studierende, Gesellschaft). Durch die Interaktion und das kommunikative Wechselspiel dieser Akteur/innen entstehen immer wieder Formen von sozialer Ordnung innerhalb qualitativer Sozialforschung und daraus Entwicklungen, die manchmal lange, manchmal kurz überleben. [4]

Diese Entwicklungen, so sehr sie erklärbar und verstehbar sind, folgen jedoch meist keiner Entfaltungslogik, sie zielen auf keine festen Punkt, auf Wahrheit oder Vernunft. Man kann sie auch nicht als Trends bezeichnen, die dauerhaft einer durchgehenden Linie folgen. Denn in diesen Entwicklungen gibt es immer wieder Löcher, Ungenauigkeiten, Widersprüche, Rücknahmen, Irrtümer, Selbsttäuschungen und Zufälle. [5]

Und Konjunkturen bestehen wieder aus Konjunkturen: Kleine Wellen sind in größere eingebettet, und diese wieder in ganz große. Konjunkturen sind ineinander geschachtelt und bilden zusammen ein eigentümliches Geflecht von kleinen, mittleren und größeren Zyklen, die einander durchdringen, aber auch beeinflussen. [6]

Obwohl bei Konjunkturen Zufälle unzweifelhaft eine Rolle spielen, folgt der Verlauf von Konjunkturen festen Regeln: Zum einen gibt es bei allen Konjunkturen eine Art Wellenbewegung des Auf und Ab, zum zweiten verläuft diese Bewegung in vier Schritten: erst kommt die Aufschwungphase (Expansion), dann die Hochkonjunktur (Boom), die in die Abschwungphase (Rezession) umschlägt und irgendwann in der Tiefphase (Depression) landet, um dann unterzugehen oder mit einer neuen Aufschwungphase einen neuen Zyklus zu beginnen. [7]

Wenn man sich einmal auf die Metaphorik des Konjunkturellen einlässt, dann fragt sich, was denn nun genau interessiert: die Konjunktur der qualitativen Sozialforschung oder die Konjunkturen der einzelnen Methoden oder der Themen innerhalb der Gesamtbewegung? Oder interessiert auch das Muster hinter dem Auf und Ab oder gar der Grund für die Wellenbewegung, also deren Ursache? [8]

\section{Erste Wertung}

Im Weiteren möchte ich mich mit der deutschsprachigen qualitativen Sozialforschung, deren Konjunktur und deren Konjunkturen beschäftigen². Diese möchte ich aus soziologischer genauer: aus wissenssoziologischer Sicht

2 Weil das so ist, werde ich auf die Darstellung der Entwicklung der qualitativen Sozialforschung in anderen Sprachräumen verzichten. Wer sagt, dass es sinnvoll ist, die deutsche Entwicklung auch als Teil der internationalen Entwicklung zu begreifen, hat Recht. Wer sagt, dass diejenigen, die nur die deutsche Entwicklung der qualitative Sozialforschung behandeln, "Nationalist/innen" seien (so Reiner WINTER beim Berliner Methodentreffen 2009), der weiß nicht, was er sagt. Genauso wenig sind diejenigen, welche nur die Entwicklung im englischsprachigen Raum (USA, Kanada, England) betrachten, deshalb Imperialist/innen, nur weil die Entwicklung im französisch-, spanisch- und portugiesischen Sprachraum nicht mit behandelt wird - und auch nicht die in Indien, Afrika, China und Australien. Man kann nicht wirklich dafür kritisieren, dass sich jemand auf seinen/ihren Gegenstand konzentriert. Man kann sich vielleicht einen anderen Gegenstand wünschen. Aber das wäre ein anderer Beitrag. 
nachzeichnen und das mit zwei Zielen: Zum Ersten geht es mir darum, die Konjunkturen der qualitativen Sozialforschung, ihrer wesentlichen Bestandteile und Themen zu rekonstruieren - zumindest in ihren wichtigsten Entwicklungen; zum Zweiten geht es mir darum, nach einem Muster "hinter" diesen Konjunkturen zu suchen. Ich will also das Feld der qualitativen Verfahren ein wenig wissenssoziologisch reflektieren. Mich interessieren dabei Fragen wie: Weshalb kommen bestimmte methodische Ansätze "hoch" (Diskurs) bzw. "nicht hoch" (Metaphern), weshalb verschwinden bestimmte Ansätze (kritische), weshalb "hypen" andere (Grounded-Theory-Methodologie)? Zu Beginn möchte ich mich jedoch mit der Konjunktur der qualitativen Forschung ganz allgemein beschäftigen. [9]

\section{Die Konjunktur der qualitativen Forschung}

\subsection{Erfolgreich oder nicht?}

Ohne Zweifel sind die Forscher und Forscherinnen, die bei ihrer Arbeit qualitative Methoden verwenden, in den letzten Jahren in Deutschland recht erfolgreich gewesen. Allerdings gilt dieser Befund nicht für jedes Fach in gleichem Maße: Für die Soziologie und die Pädagogik gilt er mehr, für die Psychologie, die Politikwissenschaft, die Kommunikationswissenschaft, die Medienwissenschaft und die Textwissenschaften gilt er teils erheblich weniger. Dennoch: auch wenn in der Mehrzahl der kulturwissenschaftlichen Fächer die qualitative Forschung immer noch mehr als Aschenputtel und weniger als Prinzessin behandelt wird, ist sie in Deutschland normal geworden. Sie hat sich sogar recht stark institutionalisiert, bedenkt man, dass die deutsche qualitative Sozialforschung sich vor etwa 40 Jahren erstmals wieder (als Reimport aus den USA) bemerkbar gemacht hat. [10]

Viele, so auch Ronald HITZLER, sind vom Erfolg der Qualitativen überzeugt, und in einem Beitrag weist er auf ein Kriterium hin, das allerdings nur für die Soziologie gilt: Demnach zeigen die bewilligten DFG-Anträge der letzten Jahre und auch die zur Zeit einflussreichen Personen in der DGS, dass qualitative Arbeiten keineswegs Karrierechancen beeinträchtigen (HITZLER 2007). Und in Ergänzung dieser Sicht könnte man sagen, dass es mehr als eine Fußnote in der Geschichte der qualitativen Forschung wert ist, wenn seit Februar 2007 zeitgleich die Präsidenten der Deutschen, Österreichischen und Schweizerischen Gesellschaft für Soziologie Vertreter der qualitativen Forschung sind: HansGoerg SOEFFNER, DGS; Christian FLECK, ÖGS; Christoph MAEDER, SGS. [11]

Man kann allerdings die Daten zur Entwicklung der qualitativen Forschung auch anders deuten. Demnach ist qualitative Forschung nicht wirklich erfolgreich: Das, was als Erfolg gewertet wird (hohe Nachfrage nach Unterweisung), sei in dem institutionellen Misserfolg der Qualitativen, also der "fehlenden Verankerung von qualitativer Forschung in der universitären Ausbildung" (MEY 2007) begründet. Das Fehlen von spezifischen Lehrstühlen und Ausbildungsangeboten sei zu beklagen (MEY 2007). Bei Ausschreibungen und bei Neubesetzungen gebe es so nicht ein Mehr an qualitativer Forschung, sondern ein Weniger. In Bezug auf 
Geld- und Personalressourcen sei qualitative Forschung ein Zwerg (ALLOLIONÄCKE \& van OORSCHOT 2007). Statt einer Institutionalisierung sei eine massive De-Institutionalisierung festzustellen (SCHREIER 2007). [12]

Werden die beiden Beobachtungen von dem Erfolg und dem Misserfolg zusammen betrachtet, verzeitlicht und zu einem Muster zusammengefügt, dann kann man sagen, dass die qualitative Forschung zwar noch boomt, aber die ersten Zeichen des Abschwungs sichtbar werden. Manches deutet auf einen erneuten Bedeutungsgewinn der quantitativen Forschung hin - einerseits aktiv, weil die quantitative Forschung wieder mehr nachgefragt wird, andererseits passiv, weil die qualitative Forschung weniger Unterstützung an den Universitäten und Forschungsinstituten findet. Die Hauptgründe hierfür sind m.E. nicht in der Kultur der Gesellschaft oder dem Markt zu suchen, sondern in der Hochschulreform und der Vergabepolitik der Drittmittelgeber. Die Quantitativen sind wieder auf dem Vormarsch, weil zum einen für die neuen Bachelor- und Masterstudiengänge die Ausbildung in den qualitativen Verfahren zu arbeits- und zeitintensiv ist. Die Hochschulreform gefährdet somit grundsätzlich die Bedingungen zur Vermittlung der Fähigkeiten für qualitative Forschung. Zum anderen zählt bei den Drittmittelgebern zu Zeiten sehr knapper Mittel wieder zunehmend die Rechenhaftigkeit von Forschung und die "Belastbarkeit" der Daten und Methoden. Auch dies bewirkt die Wiederkehr der quantitativen Forschung. [13]

\subsection{Kanonisierung bei gleichzeitiger Zersplitterung}

Der institutionelle, publizistische und auch kommerzielle Erfolg der Qualitativen hat eine eigene und teils widersprüchliche Entwicklung in Gang gebracht: nämlich die verstärkte Kanonisierung der Methoden bei gleichzeitiger Zersplitterung und Beliebigkeit. Zum einen versuchen vor allem die Protagonist/innen der etablierten qualitativen Verfahren, eine verbindliche Vorgabe über die theoretischen Prämissen und die Interpretationspraktiken zu machen, zum anderen "stricken" sich immer mehr Forscher und Forscherinnen in Anlehnung und in Ausbeutung bereits eingeführter Methoden ihre eigenen Rechtfertigungen und Deutungspraktiken. Die ersten will ich elaborierte Methoden nennen, die zweiten Ad-hoc-Methoden. [14]

Elaborierte qualitative Methoden - und das kennzeichnet sie - entwickeln vorab eine mehr oder weniger explizite Grundlagentheorie, die sich sowohl auf Wahrnehmen und Deuten bezieht als auch auf die in Betracht kommenden Gegenstände. Sie sind der Selbstreflexion grundsätzlich verpflichtet und zugleich darum bemüht, die eigene theoretische und methodische Arbeit immer wieder in die Forschung mit einzubeziehen. Zumindest das Letzte ist in der Debatte der Qualitativen nicht immer unstrittig. Zu diesen elaborierten qualitativen Methoden rechne ich zur Zeit vor allem die Grounded-Theory-Methodologie, das narrative Interview, die Ethnografie, die Konversations- und Gattungsanalyse, die objektive Hermeneutik, die dokumentarische Methode, die Diskursanalyse und die hermeneutische Wissenssoziologie. [15] 
Wer dagegen ohne vorab entworfene Grundlagentheorie die Forschungsarbeit beginnt, liefert sich (meist ohne dass es von den Forschenden selbst wahrgenommen wird) den eigenen Alltagstheorien über den Gegenstand aus dem eigenen Common Sense: Statt kontrollierter und reflektierter Erkenntnisse liefern nicht-bewusste, meist ad hoc entworfene Vor-Urteile die relevanten Ansichten über die noch zu untersuchenden Gegenstände und verlängern sie auf diese Weise. Typisch für dieses Vorgehen ist meist die Ansicht, die bislang entwickelten Methoden seien neutrale "Tools", die sich je nach Geschmack und Arbeitszeit beliebig für jede Fragestellung nutzen lassen. Weil sich hier die Auswahl der Verfahren der Datenerhebung und Datenauswertung eher aus situativen Erwägungen ergibt, möchte ich diese Verfahrensweisen als qualitative Ad-hoc-Forschung bezeichnen. [16]

MRUCK (2007) macht darauf aufmerksam, dass qualitative Forschung sich von Disziplin zu Disziplin und von Land zu Land wesentlich unterscheidet (ähnlich auch FLICK 2007 und SCHREIER 2007) - vor allem, wenn man nicht nur die USA, England und Frankreich mit einbezieht, sondern auch Arbeiten aus Lateinamerika, Asien oder Afrika. Die Lage sei nicht wirklich übersichtlich. Alle unter einen Hut oder auf einen Nenner zu bringen (= gleiche Standards und Kriterien), halten FLICK und MRUCK für aussichtslos. Allerdings sei die deutsche Forschungspraxis und Debatte vom internationalen Diskurs zu großen Teilen abgekoppelt (FLICK 2007). [17]

Man kann das als bunte Vielfalt deuten ("Lasst tausend Blumen blühen"), als Zersplitterung (Chaos, Zerfall), als Diversifikation (also die Verbreiterung des Angebots), als Differenzierung (also als bessere Anpassung an den Gegenstand) oder als Trennung von Arbeitswelten. Mir scheint es auf Diversifikation und eine Trennung von Arbeitswelten hinauszulaufen. Man spezialisiert sich auf bestimmte Märkte und entwickelt vor dieser Spezialisierung nur noch das, was man dort benötigt. [18]

\section{Konjunkturen der methodischen Ansätze}

Manche Ansätze sind bereits vergessen: Oral history, Aktionsforschung und kritische Sozialforschung sind vergangen und die Diskursanalyse ist gekommen. Dauerbrenner sind das Experteninterview und die Methodologie der Grounded Theory. Die Metaphernanalyse kommt nicht so richtig auf die Beine. Bild- und Videoanalyse und der Artefaktforschung wird die Zukunft gehören. Von der kommunikativen Validierung spricht heute fast niemand mehr, sondern für fast alle sind Triangulation, Mixed Methods und computergestützte Auswertung Themen, mit denen man sich auseinandersetzen muss. Vor allem an der computergestützten Auswertung kommt keine/r vorbei, und zugleich scheiden sich an ihr die Geister. [19]

Der Boom der comptergestützten qualitativen Sozialforschung ist unübersehbar: Allerorts wird der Eindruck verbreitet, als könne man CAQDA (Computer Assisted Qualitative Data Analysis) als neutrales Tool benutzen, das in (fast) allen Wissenschaftsdisziplinen, bei allen Verfahren und bei allen Textsorten hilfreich 
ist, da es die Daten vor allem verwaltet und ordnet und bei der Kategorienzuweisung und -verbindung die Arbeit erleichtert - also Ordnung schafft. Dass sie jedoch kein neutrales Werkzeug ist, da nämlich jede Art des Ordnens, also auch die mit Papier und Bleistift, eine auch theoretisch zu rechtfertigende Form der Theoriekonstruktion ist, das ist zwar vielen klar (KUCKARTZ 2005), aber nur sehr selten wird es explizit gesagt: Kategorien fallen weder vom Himmel, noch werden sie von der Software geliefert, sondern sie müssen mittels eigenständiger Deutungstätigkeiten erarbeitet werden. Dennoch entsteht der Eindruck, als sähe das die Forschungspraxis mitunter völlig anders. [20]

\section{Konjunkturen der Themen}

Was sind die aktuell relevanten Themen innerhalb der qualitativen Sozialforschung? Hier die wichtigsten mit kurzen Kommentaren: [21]

\subsection{Gültigkeit}

Das Problem der Gültigkeit war und ist für die qualitative Sozialforschung notorisch. Darüber ist schon viel gesagt und geschrieben worden, sodass wenig Neues hinzugefügt werden kann. Nur so viel: Ohne Zweifel ist die intersubjektive Nachvollziehbarkeit der zentrale Wert, wenn es darum geht, die Güte einer qualitativen Forschung zu beurteilen. Dies bezieht sie auch darauf, dass die Anzahl der Fälle, die Auswahl und Gestaltung der Methoden und ihre Abstimmung auf den zu untersuchenden Gegenstand von allen Kollegen und Kolleginnen, die guten Willens sind, nachvollzogen werden können. Zu dieser Nachvollziehbarkeit gehört, dass alle wichtigen Entscheidungen in den Forschungsberichten dargestellt werden und im Falle eines Falles auch nachgeprüft werden können. Auch wenn es noch eine Vielzahl von ethischen und rechtlichen Problemen gibt, scheint mir kein Weg daran vorbeizugehen, dass qualitativ arbeitende Forscher und Forscherinnen ihre Daten in geeigneter Form lokal oder zentral archivieren und für Sekundäranalysen zur Verfügung stellen (siehe zum Problem der Archivierung den guten Diskussionsband von BERGMANN \& EBERLE 2005). Zu dieser Nachvollziehbarkeit gehört sicherlich auch, dass die angewandten Methoden, gerade weil in der qualitativen Sozialforschung das Feingefühl von größter Bedeutung ist, nicht auf die Person eines bestimmten Forschers oder einer bestimmten Forscherin angewiesen sind, sondern von jedem/jeder eingesetzt werden können und zu einem vergleichbaren Ergebnis führen. Ohne eine solche Kanonisierung der einzelnen Methoden ist die qualitative Sozialforschung nicht zukunftsfähig oder genauer: sie überlebt den einzelnen Forscher bzw. die Forscherin nicht. [22]

Will man die Güte qualitativer Forschung im wissenschaftlichen Diskurs (aber vor allem auch im Diskurs mit potenziellen Bewerter/innen) verteidigungsfähig machen, dann gelingt dies heute keinesfalls mehr durch die Berufung auf die Autorität verstorbener Säulenheiliger der Wissenschaft, auch nicht durch den empiriefreien Einsatz wissenschaftlicher Vernunft und ebenfalls nicht durch die Unterstellung persönlicher Hellsichtigkeit. Stattdessen lässt sich die Güte von Aussagen nur über empirische Forschung rechtfertigen und deren Güte 
wiederum über spezifische (nach Gesellschaft, Zeit und Fachgebiet variierende) Standards der Qualitätssicherung. Letztere werden sich jedoch dabei (zumindest im westlichen Wissenschaftsprogramm) auf die Fragen der Zuverlässigkeit und der Repräsentativität der Datenerhebung und auf die Gültigkeit der Generalisierung beziehen müssen - will man in dem Wettbewerb um ökonomisches Forschungskapital im Spiel bleiben. [23]

Kann bei der Bewältigung dieser nicht einfachen Aufgabe die qualitative Forschung (im Allgemeinen) unter Zugrundelegung eines (unreflektierten) Realismus solche Verfahren favorisieren, die versprechen, näher an der "Wirklichkeit" zu sein, so kann dieses Kriterium innerhalb einer reflexiven Sozialforschung so nicht gelten - hat sie sich doch von der Möglichkeit der "Wirklichkeitsansicht" verabschiedet, allerdings verbunden mit der Hoffnung, empirische Forschung und wissenschaftlicher Diskurs produzierten, wenn schon keine guten, dann jedoch bessere Einsichten. [24]

Eine qualitativ verfahrende Datenanalyse, deren Validität sowohl durch den Datenbezug als auch durch konkurrierende Lesartenkonstruktionen und den wissenschaftlichen Diskurs gesichert werden soll, hat notwendigerweise zur Voraussetzung, dass mehrere ausgebildete Wissenschaftler/innen das Material unabhängig voneinander interpretieren und ihre Ergebnisse immer wieder einer wissenschaftlichen Kritik aussetzen. Die Sicherung der wissenschaftlichen Ressourcen, um eine solche Überprüfung von Lesarten, Hypothesen und theoretischen Verallgemeinerungen vorzunehmen, trägt dabei nicht unwesentlich zur Erhaltung selbstverständlicher Standards, wissenschaftlicher Anforderungen an die Validität von Untersuchungen bei - was bedeutet, dass die qualitative Forschung nicht weiter auf den Schultern von Einzelkämpfer/innen ruhen darf, sondern die kooperative und konkurrierende Teamarbeit muss selbstverständlicher Standard werden. [25]

Nur wenn die Standards wissenschaftlicher Güteprüfung in der qualitativen Forschung fest etabliert und auch weiter ausdifferenziert werden, hat dieses Forschungsprogramm unter den aktuellen Bedingungen eine Chance, auf dem Markt zu bleiben - was im Übrigen nicht heißt, dass in irgendeiner Weise eine "Evidence-Based Pratice" (vgl. McNEECE \& THYER 2004) angestrebt werden sollte. Gelingt eine solche Ausarbeitung, Abwägung und Kanonisierung der Standards in absehbarer Zeit nicht, dann werden qualitative Studien zwar in den Medien ein gewisses Echo finden, aber ansonsten werden sie eine gute Chance haben, bedeutungslos zu werden: Der qualitativ ausgebildete Nachwuchs wird schwerer in einen Beruf finden, qualitative Projekte werden minimal oder gar nicht mehr finanziert werden - was schlussendlich zur Marginalisierung dieser Forschungstradition führen wird. [26]

\subsection{Festschreibung von Offenheit}

Das "Offenheitspostulat" qualitativer Forschung wird präzisiert - darüber scheint Einigkeit zu herrschen. Offenheit bedeutet in dieser Präzisierung, nicht vorweg das untersuchte Feld mit fixen Hypothesen zu überziehen, es bedeutet, offen für 
das möglicherweise Neue zu sein und zu bleiben. Offenheit in der qualitativen Forschung heißt jedoch nicht, dass Forscher/innen im Hinblick auf den untersuchten Gegenstand, die Methodologie und die Methode, bewusst "dumm" bleiben, sich vorab also nicht informieren. [27]

In seinem viel gerühmten Artikel hat Ronald HITZLER ausdrücklich von der "künstlichen Dummheit" gesprochen, nicht von der tatsächlichen (HITZLER 1991). Künstliche Dummheit weiß viel, weil sie sich vorher umfassend informiert hat, was sie wie untersuchen will, klammert aber die Gültigkeit dieses Wissens aus und bleibt so offen für Altes und Neues (s. auch REICHERTZ 2003). Künstliche Dummheit kennt auch den State of the Art zum Gegenstand, zur Methode und zur Methodologie. Mit dieser systematischen Vergrößerung des eigenen Wissens vergrößert man zugleich den verfügbaren Raum der guten Gründe, etwas zu tun - und erspart sich dann später auch die leidigen Diskussionen über das, was als "akzeptiert" gilt. Das ist hilfreich bei der Interpretation und auch bei der späteren Theoriebildung. Wer sich vor der Arbeit nicht schlau macht, wird außer den eigenen Vor-Urteilen nichts finden, läuft zudem Gefahr, die Kuckucksuhr neu zu erfinden und darf sich über das Kopfschütteln der Scientific Community nicht wundern. [28]

\subsection{Das Allgemeine oder das Besondere oder was?}

Wenn ich es richtig sehe, beschränken sich nur mehr sehr wenige qualitativ arbeitende Forscher/innen auf die Deskription des Einzelfalles. Allen geht es mittlerweile auch um das Muster, das verbindet, das Muster, das verständlich macht, das erklärt. Ich sehe also keinen generellen Verzicht der Qualitativen auf Erklären - wie das manche behaupten und (wie sie sagen) auch fürchten (KLÜVER 2007). Einzuräumen ist allerdings, dass innerhalb qualitativer Forschung "Erklären" nicht mehr mit einem einfachen Determinismus verbunden werden kann, sondern nur noch mit Wahrscheinlichkeiten. [29]

\subsection{Triangulation}

Auch über die Triangulation wird viel diskutiert und vor allem: Mit ihr verbinden sich viele, auch trügerische Hoffnungen. Manche versprechen sich von ihr Genauigkeit, andere gar Objektivität - was sie tatsächlich bringt, ist Multiperspektivität. [30]

Denn die Triangulation will die Erkenntnis von der wissenschaftlichen Perspektivität produktiv nutzen, indem sie als Gütegarant eine als positiv deklarierte Multi-Perspektivität anstrebt. Qualitative Verfahren werden mit quantitativen ergänzt, die Feldstudie mit Interviews und Fragebogen, die Interaktionsanalyse mit Experiment und Beobachtung. Die Grundidee (bzw. die zugrunde gelegte Metapher) dieser Strategie ist der Geometrie entlehnt: Um einen nicht erreichbaren Punkt (Erkenntnis) zu bestimmen, peile ich diesen Punkt von zwei (oder mehr) bekannten Perspektiven (Methoden) aus an, bestimme das Verhältnis der bekannten Perspektiven zueinander und deren "Winkel" zum angepeilten Punkt, und kann dann mithilfe trigonometrischer Berechnungen den 
unbekannten Punkt bestimmen. Bei der Methoden-Triangulation geht es also nicht darum, die Perspektivität zu leugnen, sondern sie zum Programm zu erheben. Am Ziel angekommen ist man, wenn alles trotz seiner Vielfältigkeit ein einheitliches Bild ergibt. [31]

Das Triangulationsprogramm kann noch erweitert werden: So kann sich zu der Triangulation der Methoden noch die Triangulation der Daten und der Interpret/innen gesellen. Das Ergebnis ist immer das gleiche: Perspektiven und Perspektivität werden sichtbar, der untersuchte Gegenstandsbereich zeigt sich von vielen Punkten - aber Wahrheit erzielt man so nicht. [32]

\subsection{Mixed Methods}

Es ist friedlich geworden im Feld der Sozialforschung. Fast niemand zieht heute noch aus, um die Quantitativen aus dem Felde zu schlagen. Vor allem in der Soziologie und Pädagogik sind die Zeiten vorbei und fast schon vergessen. Die Zeit der metatheoretischen und paradigmatischen Abgrenzungsbemühungen gegenüber quantifizierenden, nomologisch-deduktiv verfahrenden Ansätzen scheint vorerst vorüber. Die "lange erstarrten Fronten [sind] (...) - unverkennbar in Bewegung geraten" (ESSER 2007, S.351). [33]

Selbst Vertreter/innen der quantitativen Forschung scheinen auf einen Konsenskurs eingeschwenkt zu sein. Oft wird neuerdings (in beiden Gruppen) von zwei Forschungsstrategien gesprochen, die beide ihre Berechtigung hätten, es käme halt auf die jeweilige Frage und das jeweilige Ziel der Forschung an, ob die eine oder die andere Strategie einzusetzen sei. Zudem solle eine Streitkultur erblühen, die über die Stärken und Schwächen der Methoden in Konkurrenz tritt und so den Besten bzw. die beste Methode überleben lässt (SCHREIER 2007). Andere propagieren bereits die Mixed Methods (z.B. ERZBERGER 1998; KELLE \& ERZBERGER 2000; KELLE 2008) - im Übrigen mit beachtlichem Erfolg. [34]

Ohne Zweifel macht es Sinn, dann quantitative mit qualitativen Verfahren (und vice versa) zu verbinden, wenn Forschungsfrage und Forschungsziel das rechtfertigen. Denn vieles ist beiden Gruppen gemein. In beiden Gruppen arbeiten Forscher und Forscherinnen offen, kommunikativ, flexibel, intersubjektiv und reproduzierbar. In beiden Gruppen gibt es welche, die sich auf Aussagen über Zusammenhänge kleiner Reichweite beschränken, und andere, die zudem noch Zusammenhänge mittlerer und großer Reichweite erklären wollen. In beiden Gruppen finden sich Forscher/innen, welche die Mikroperspektive, und andere, welche die Makroperspektive bevorzugen. Manchmal liefern bei Forschungsarbeiten die Quantitativen die Hypothesen, manchmal tun das die Qualitativen, und manchmal überprüfen die Quantitativen, manchmal die Qualitativen. [35]

Und natürlich ruht jede quantitative Untersuchung auf einer qualitativen Basis und muss sie im Verlauf der Arbeit immer wieder interpretieren - weshalb in quantitativen Untersuchungen immer und notwendigerweise mit den Prämissen qualitativer Forschung gearbeitet wird. Und umgekehrt ruht jede qualitative 
Untersuchung auf einer quantitativen Basis (z.B. dann, wenn sie "Normalität" bestimmen will) und muss im Verlauf der Arbeit immer wieder die Relevanz, die Wichtigkeit, die Häufigkeit "intuitiv" ermitteln - weshalb in qualitativen Untersuchungen immer und notwendigerweise mit den Prämissen quantitativer Forschung gearbeitet wird. [36]

In der konkreten Forschung durchdringen sich also das Feststellen von Häufigkeiten und die Ausdeutung von Sachverhalten - und zwar in jeder Phase der Forschung. Es gibt sie nicht, die klare Trennung der quantitativen und qualitativen Forschung entlang von bestimmten Merkmalen, Phasen, Perspektiven oder Reichweitenansprüchen: Gründe genug, darauf zu hoffen, dass es auch eine echte Verbindung der beiden Methodenverständnisse geben könnte. [37]

Was die beiden Forschungsrichtungen allerdings trennt, das ist der Umstand, dass jede Richtung eine eigene Kultur besitzt, und dass diese Kulturen nicht so viele inhaltliche Gemeinsamkeiten aufweisen. Das gilt insbesondere, wenn man im Anschluss an Hans-Georg SOEFFNER (1988, S.12) unter "Kultur" jenen "Bedeutungsrahmen [versteht], in dem Ereignisse, Dinge, Handlungen, Motive, Institutionen und gesellschaftliche Prozesse dem Verstehen zugänglich, verständlich beschreibbar und darstellbar sind". Obwohl manche sprachlichen Formulierungen ähnlich klingen, ist eine Reise von der qualitativen Forschung zur quantitativen nicht mit einer Reise von Dortmund nach Essen, sondern mit einer von Dortmund nach Detroit zu vergleichen: Ein Ozean trennt die beiden Kulturen - um eine alte Idee und Metaphorik von SNOW (1965) aufzugreifen. Quantitative und qualitative Forschung sind nicht nur durch die Methoden getrennt, sondern vor allem und wesentlich: durch die Kultur, deren Ausdruck die Methoden sind. [38]

\section{Weisen die Konjunkturen ein Muster auf? ${ }^{3}$}

Bei aller Widersprüchlichkeit und aller Gleichzeitigkeit des Widersprüchlichen lassen sich doch aus der Distanz größere Bewegungen erkennen, d.h hinter den kleinen ("jahreszeitlichen") Konjunkturschwankungen finden sich große Konjunkturwellen. Nicht alle diese Wellenbewegungen, die man im Übrigen nicht mit Trends verwechseln darf, sind klar, und nicht alle befinden sich in allen Disziplinen in der gleichen Entwicklungsphase. Manche stehen erst am Anfang, andere sind in der Boomphase, andere in der Rezession. Aus meiner Sicht sind das vor allem folgende Bewegungen: [39]

3 Die hier vorgetragene Einschätzung der aktuellen Entwicklung der qualitativen Sozialforschung beruht nicht auf einer eigenen empirischen Untersuchung mit Fragebogen oder breit gestreuten Interviews. Auch resultiert sie nicht aus einer systematischen Sichtung der Literatur. Dies deshalb, weil es hierzu (noch) keine Literatur oder eine wissenschaftssoziologische Studie gibt. Meine Einschätzung beruht also im Wesentlichen auf meiner "Mitspielkompetenz" und meiner Kenntnis dieses Feldes. Als Mitglied der zweiten Generation qualitativer Sozialforschung habe ich das Auf und Ab von Beginn an miterlebt, dann auch (in kleinen Teilen) mitgestaltet und dabei die Wandlungsprozesse an der eigenen Forschungspraxis und die der anderen miterleben können. Natürlich sind in meine Einschätzung auch die vielen Diskussionen mit Kolleginnen und Kollegen (auf Tagungen und vor allem: nach Tagungen) eingegangen, bei denen wir uns über die Entwicklung und die aktuelle Praxis der qualitativen Forschung ausgetauscht haben. Die hier vorgetragene Einschätzung ist also im wahrsten Sinne des Wortes eine Einschätzung (eines Feldakteurs) - nicht mehr, aber auch nicht weniger. 


\subsection{Auf der Ebene des Forschungsgegenstandes}

1. Vom subjektiven Sinn zur gesellschaftlichen Bedeutung

2. Von der Intention zur Praxis

3. Vom Subjekt über den Diskurs zur Praxis

4. Von der symbolischen Interaktion zur symbolischen Ordnung

5. Von der Freiheit der Einzelnen zur Macht von kollektiven Praktiken

6. Vom Text zum stehenden und laufenden Bild [40]

\subsection{Auf der Ebene der Forschungspraxis}

1. Vom Einzelfall zur größeren Zahl, dem Fallvergleich und der Sekundäranalyse

2. Von der Beobachtung zu belastbaren Daten

3. Von der Deutung zur Auswertung

4. Von der Handarbeit zur Maschinenarbeit (PC, Kamera, Diktiergerät, Software)

5. Vom sozialen Gruppenprozess zur computergestützten Einzelarbeit

6. Von der Wetware (Kultur und Wissen) zur Software (Programm und Regel)

7. Von teuer und langsam zu "quick and cheap"

8. Vom gemeinsamen Aufbruch zur erbitterten Konkurrenz

9. Von der theoretischen und methodologischen Debatte zur praktischen Arbeit am Forschungsgegenstand [41]

\subsection{Auf der Ebene der Legitimation von Forschung}

1. Von der persönlichen Evidenz zur Rechtfertigung mit Gütesiegeln (Rechenhaftigkeit, Verfahren, Evaluation)

2. Vom Subjektiven zum Objektiven

3. Von der Deutung zur Rekonstruktion

4. Vom Forschungssubjekt zum Forschungsverfahren

5. Vom charismatischen "Führer" zur kanonisierten Methode - Veralltäglichung und Bürokratisierung des Charismas

6. Von der Aufklärung vor Ort zur Zeitdiagnose und Sozial- und Gesellschaftstheorie mittlerer Reichweite [42]

\subsection{Auf der Ebene der Adressat/innen von qualitativer Forschung}

1. Von der Forschung für Betroffene oder die Gesellschaft zur Forschung für bestimmte (wirtschaftliche, politische) Interessen

2. Vom Kampf gegen die Quantitativen zur fragen- und projektbezogenen Kooperation

3. Von Forscher/innen und Studierenden zu Drittmittelgebern aus Hochschule, Politik, Verwaltung, Medien und Wirtschaft 
4. Von wissenschaftlichen Kolleg/innen zu wissenschaftlichen wie außerwissenschaftlichen "Kunden"

5. Von der Reputation zum ökonomischen Erfolg [43]

\section{Was bewegt die verschiedenen Konjunkturen?}

Man kann die hier beschriebene Deutung der Entwicklung qualitativer Sozialforschung aber noch eine Stufe weitertreiben. So kann man versuchen, das Muster hinter diesen aufgelisteten einzelnen Entwicklungen zu "erraten" - also erste wissenschaftssoziologische Konzeptionalisierungen einer Entwicklungstheorie qualitativer Sozialforschung vorzunehmen. Dabei lassen sich aus meiner Sicht drei wesentliche Großentwicklungen feststellen": [44]

\subsection{Veralltäglichung des Charismas}

Die qualitativen Verfahren entstanden nicht aus dem Nichts, sie sind nicht vom Himmel gefallen, sondern Ergebnis eines allgemeinen (Wege zur Erkenntnis) und speziellen (quantitativ vs. qualitativ) gesellschaftlichen Aushandlungsprozesses. Das Wiederaufleben der qualitativen Verfahren, einerseits als Reimport aus den USA, andererseits durch die Auseinandersetzung mit dem Kritischen Rationalismus, wurde vor allem innerhalb einer Generation von verschiedenen "Charismatikern" (in Frontstellung gegen die quantitativen Verfahren - siehe KNOBLAUCH 2007) geleistet und auch durchgesetzt (z.B. SCHÜTZE, OEVERMANN, SOEFFNER, LUCKMANN, LEITHÄUSER). Es ist m.E. gewinnbringend, WEBERs Charismatheorie auf die Entwicklung der qualitativen Forschung anzuwenden und zu prüfen, wie das Charisma in den einzelnen Traditionen veralltäglicht und verwaltet wurde und wird (siehe auch SCHNETTLER 2007). Das eine, was die Konjunkturen bewegte, war aus meiner Sicht also die Veralltäglichung des Charismas der "Gründergestalten" und die damit einhergehende Profanisierung und Bürokratisierung der qualitativen Sozialforschung. [45]

\subsection{Vom Reputationsmarkt zum ökonomischen Markt}

Mir scheint, als würde sich das Feld der Qualitativen spalten, als würden Trennungsprozesse beobachtbar sein: Einige schreiben immer noch für die Kolleg/innen und sehen die Akteur/innen oder die Gesellschaft als ihre Klientel an, andere schreiben klammheimlich oder offen für die Politik, die Hochschule, die Medien oder die Wirtschaft. Sie schreiben in deren Sprache, verwenden deren Argumente und Sichtweisen und explizieren das eigenen Tun nur so weit, wie es für die Abnehmer anschlussfähig ist. Und die Abnehmer sind im

4 Eingebettet ist die Entwicklung der qualitativen Sozialforschung in einen umfassenderen sozialen Prozess der Neubewertung der verschiedenen Wissenschaften: "Seit den 90er Jahren verschieben sich die Gewichte zwischen den Disziplinen dramatisch: Natur-, Technik- und Lebenswissenschaften erringen Dominanz. In der neu etablierten Diskursformation Exzellenzförderung, Wettbewerb, Elitebildung erleiden die Geistes- und Sozialwissenschaften einen vor dem Hintergrund in der Blüte in den 60er und 70er Jahren umso drastischer erfahrenen Niedergang" (SCHEFFER \& SCHMIDT 2009, S.293f.). 
Wesentlichen "Kunden", die direkt oder indirekt Forschung finanzieren. Das ist keineswegs ehrenrührig - es verändert aber die Forschungsarbeit. [46]

\subsection{Abschied von Therapie und Kritik}

Qualitative Forschung war zu Beginn eine (Gegen-) Bewegung. Ihr wohnte ein kritischer Impuls inne. Es ging anfangs nicht nur gegen eine den Sinn vernachlässigende und die Zahlen bevorzugende Wissenschaft, sondern auch gegen eine Wissenschaft als Beruf, die sich aller Wertung enthalten wollte. Ein wichtiger Kristallisationspunkt dieser Debatte war der zweiter Werturteilsstreit Ende der 1960er Jahre, also die Debatte zwischen POPPER, ADORNO, HABERMAS, PILOT, DAHRENDORF und ALBERT (siehe ADORNO et al. 1972) darüber, was die gesellschaftliche Aufgabe der Soziologie zu sein habe. Ein beachtlicher Teil der Qualitativen hatte die Kritik des Bestehenden und die Therapie des Kranken auf ihre Fahnen geschrieben. Die starke Subjektorientierung, also die Konzentration auf das wollende und handelnde Subjekt und die damit einhergehende Phantasie, das Subjekt sei der wahre und letzte Verursacher sozialen Handelns, machte dann die Forschung wesentlich herrschaftsneutral, denn der Blick wurde von den gesellschaftlichen Ordnungen abgezogen und auf die Intentionen der Subjekte gerichtet. Dem ist so. Aus meiner Sicht hat die qualitative Forschung im Bemühen um die Anerkennung als wissenschaftliches Verfahren (auch in den Augen der Quantitativen) mit Max WEBER auf die Wertfreiheit gesetzt. Diese Strategie war erfolgreich. Der Preis für den Erfolg war der weitgehende Verzicht auf Kritik und Therapie. Dem muss aber nicht so sein. [47]

Jede Wissenschaft, also auch die qualitative Sozialforschung, lebt in der jeweiligen Gesellschaft und lebt von ihr (finanziell wie inhaltlich). Als solche steht sie nicht außerhalb der Gesellschaft, sondern ist einerseits Ergebnis gesellschaftlicher Entwicklungen und gestaltet andererseits immer auch Gesellschaft durch ihre Arbeit und die Publikation ihrer Ergebnisse mit. Jede Wissenschaft, also auch die qualitative Sozialforschung, kommuniziert mit der Gesellschaft, die sie erforscht und über die sie schreibt. Und: sie gibt nicht nur, sondern bevor sie anfängt, hat sie schon sehr viel erhalten. Wissenschaft ist auch Ausdruck der Kultur einer Gesellschaft. Kurz: Wissenschaft hat stets auch gesagt, was Subjekte, die Gesellschaft, Normen, Werte, Biografien sind und sein sollten und hat dabei vor allem eigene Wertvorstellungen und Interessen vertreten. Die Wissenschaft und natürlich auch die qualitative Sozialforschung waren von Beginn an Partei und nicht uninteressierte Beobachter im Elfenbeinturm. Als solche hatten sie auch, ob sie das wollten oder nicht, Verantwortung für die Gesellschaft, für die sie schrieben. Der sollten sie sich wieder bewusst werden. [48] 


\section{Literatur}

Adorno, Theodor W.; Dahrendorf, Ralf; Pilot, Harald; Albert, Hans; Habermas, Jürgen \& Popper, Karl R. (1972). Der Positivismusstreit in der deutschen Soziologie. Neuwied: Luchterhand.

Allolio-Näcke, Lars \& Jürgen van Oorschot (2007). Suchbewegungen zum Überleben der Qualitativen Sozialforschung? Erwägen - Wissen - Ethik, 18(2), 208.

Bergman, Manfred Max \& Thomas S. Eberle (Hrsg.) (2005). Qualitative Forschung, Archivierung, Sekundärnutzung: Eine Bestandsaufnahme. Forum Qualitative Sozialforschung / Forum: Qualitative Social Research, 6(2), http://www.qualitative-research.net/fqs/fqs-d/inhalt2-05-d.htm [Datum des Zugriffs: 29. Mai 2005]

Erzberger, Christian (1998). Zahlen und Wörter. Die Verbindung quantitativer und qualitativer Daten und Methoden im Forschungsprozess. Weinheim: Deutscher Studienverlag.

Esser, Hartmut (2007). Soll das denn alles (gewesen) sein? Soziale Welt, 58, 351-358.

Flick, Uwe (2007). Diversifizierung, Güte und Kultur qualitativer Sozialforschung. Erwägen Wissen - Ethik, 18(2), 222.

Hitzler, Ronald (1991). Dummheit als Methode. In Detlev Garz \& Klaus Kraimer (Hrsg.), Qualitativempirische Forschung (S.295-318). Opladen: Westdeutscher Verlag.

Hitzler, Ronald (2007). Nur Sinn macht Sinn. Erwägen - Wissen - Ethik, 18(2), 229.

Kelle, Udo \& Erzberger, Christian (2000). Qualitative und quantitative Methoden: Kein Gegensatz. In Uwe Flick, Ernst von Kardorff \& Ines Steinke (Hrsg.), Qualitative Forschung. Ein Handbuch (S.299-309). Reinbek: Rowohlt.

Kelle, Udo (2008). Die Integration qualitativer und quantitativer Methoden in der empirischen Sozialforschung: Theoretische Grundlagen und methodologische Konzepte. Wiesbaden: VS Verlag.

Klüver, Jürgen (2007). Die Überkomplexität der qualitativen Sozialforschung. Erwägen - Wissen Ethik, 18(2), 239.

Knoblauch, Hubert (2007). Die Gestaltung der qualitativen Sozialforschung aus dem Geiste des Interpretativen Paradigmas. Erwägen - Wissen - Ethik, 18(2), 242.

Kuckartz, Udo (2005). Einführung in die computergestützte Analyse qualitativer Daten. Ein Lehrbuch. Wiesbaden: VS Verlag.

Kuhn, Thomas (1976). Die Struktur wissenschaftlicher Revolutionen. Frankfurt/M.: Suhrkamp.

McNeece, C. Aaron \& Bruce A. Thyer (2004). Evidence-based practice and social work. Journal of Evidence-Based Social Work, 1(1), 7-25.

Mey, Günter (2007). Qualitative Forschung in der Lehre ... eine Leerstelle? Erwägen - Wissen Ethik, 18(2), 255.

Mruck, Katja (2007). Qualitative Forschung: Notizen aus der Übersichtlichkeit. Erwägen - Wissen Ethik, 18(2), 258.

Popper, Karl (2004). Objektive Erkenntnis. Hamburg: Hoffmann und Campe.

Reichertz, Jo (2003). Die Abduktion in der qualitativen Sozialforschung. Opladen: Leske + Budrich.

Reichertz, Jo (2007a). Qualitative Sozialforschung - Ansprüche, Prämissen, Probleme. Erwägen Wissen - Ethik, 18(2), 195-208. Mit kritischen Kommentaren von Lars Allolio-Näcke \& Jürgen van Oorschot, Georg Breidenstein, Franz Breuer, Thomas Brüsemeister, Thomas Eberle, Hannelore Faulstich-Wieland \& Peter Faulstich, Uwe Flick, Jochen Gläser \& Grit Laudel, Detlef Garz, Heiko Grunenberg, Ronald Hitzler, Gerd Jüttemann, Mechthild Kiegelmann, Dieter Kirchhöfer, Jürgen Klüver, Hubert Knoblauch, Helmut Kromrey, Thomas Loer, Morus Markard, Philipp Mayring, Wolfgang Mertens, Günter Mey, Katja Mruck, Karin Schlücker, Bernt Schnettler, Margit Schreier, Johannes Twardella, Werner Vogd, Jan Weyand und Harald Witt.

Reichertz, Jo (2007b). Qualitative Forschung auch jenseits des interpretativen Paradigmas? Erwägen - Wissen - Ethik, 18(2), 276-293.

Scheffer, Thomas \& Schmidt, Robert (2009). Soziologie als modus operandi. Soziologie, 3 , 291-306.

Schnettler, Bernt (2007). Qualitative Sozialforschung: Charisma, Schule, Betrieb. Erwägen Wissen - Ethik, 18(2), 262. 
Schreier, Margit (2007). Qualitative Sozialforschung in Zeiten von Hochschulreform und Globalisierung. Erwägen - Wissen - Ethik, 18(2), 264.

Snow, Charles P. (1965). The two cultures: And a second look. Cambridge: Cambridge University Press.

Soeffner, Hans-Georg (1988). Kulturmythos und kulturelle Realität(en). In Hans-Georg Soeffner (Hrsg.), Kultur und Alltag (S.3-20). Göttingen: Schwarz Verlag.

\section{Zum Autor}

Jo REICHERTZ, Jahrgang 1949, Studium der Germanistik, Mathematik, Soziologie und Kommunikationswissenschaft. Promotion zur Entwicklung der "Objektiven Hermeneutik", Habilitation mit einer soziologischen Feldstudie zur Arbeit der Kriminalpolizei; seit 1993 Professor für Kommunikationswissenschaft an der Universität Duisburg-Essen, Campus Essen - zuständig für die Bereiche "Strategische Kommunikation", "Qualitative Methoden", "Kommunikation in Institutionen", und "Neue Medien".

Arbeitsschwerpunkte: qualitative Sozialforschung, wissenssoziologische Text- und Bildhermeneutik, Kultursoziologie, Religionssoziologie, Medienanalyse, Mediennutzung, empirische Polizeiforschung, Werbe- und Unternehmenskommunikation.
Kontakt:

Prof. Dr. Jo Reichertz

Fachbereich Geisteswissenschaften

Universität Duisburg-Essen

Universitätsstraße 12

D-45141 Essen

Tel.: $0201183-2810$

E-Mail: jo.reichertz@uni-due.de URL: http://www.uni-essen.de/kowi/reichertz/

\section{Zitation}

Reichertz, Jo (2009). Die Konjunktur der qualitativen Sozialforschung und Konjunkturen innerhalb der qualitativen Sozialforschung [48 Absätze]. Forum Qualitative Sozialforschung / Forum: Qualitative Social Research, 10(3), Art. 30, http://nbn-resolving.de/urn:nbn:de:0114-fqs0903291. 